\title{
Effects of Nipradilol, a Nitric Oxide-Releasing $\beta$-Adrenoceptor Blocking Agent, on Phosphoenolpyruvate Carboxykinase Gene Transcription in a Rat Hepatoma Cell Line
}

\author{
Keishi Yamauchi*, Koji Nakajima, Sachiko Ikeo, Yoshihiro Nishimura, Mitsuhisa Komatsu, \\ Toru Aizawa and Kiyoshi Hashizume \\ Department of Aging Medicine and Geriatrics, Shinshu University School of Medicine, Matsumoto 390-8621, Japan
}

Received March 12, 2001 Accepted July 13, 2001

\begin{abstract}
Effects of nipradilol, a $\beta$-adrenoceptor blocker with a nitroxy moiety, on phosphoenolpyruvate carboxykinase (PEPCK) gene transcription were examined using a rat hepatoma cell line, H4IIE cells. Dexamethasone was employed as an enhancer of PEPCK gene transcription. Nipradilol, but not timolol (a $\beta$-blocker without a nitroxy moiety), attenuated PEPCK gene transcription both in the control and the dexamethasone-treated cells. The effects of nipradilol were eradicated by methylene blue (an inhibitor of cellular guanylate cyclase). Nipradilol is a unique $\beta$-blocker that suppresses PEPCK gene transcription in hepatocytes likely through liberation of nitric oxide and resultant activation of guanylate cyclase.
\end{abstract}

Keywords: Dexamethasone, Phosphoenolpyruvate carboxykinase, Nipradilol

Insulin resistance is an important etiologic abnormality in type 2 diabetes mellitus. Namely, insulin action at the insulin-target tissues such as skeletal muscle and adipose tissue as well as liver is impaired, and much of the hepatic insulin resistance is due to inappropriately increased hepatic gluconeogenesis. Glucocorticoid is a strong diabetogenic hormone that induces insulin resistance both in vitro and in vivo (1). At the level of liver, glucocorticoid increases gluconeogenesis so that it partially mimics the abnormality found in patients with diabetes.

Nipradilol, 3,3-dihydro-8-(2-hydroxy-3-isopropylamino) proxy- $2 H$-1-benzopyran, is a potent $\beta$-adrenoceptor blocking agent with nitroxy moiety (Fig. 1A), which induces vasodilatation (2) and improves glucose metabolism in vivo (3). Because nitric oxide (NO) improves glucose metabolism through vasodilatation of insulin-target tissues (4), nipradilol's antidiabetic effect can be attributed to the NO provision at the level of vasculature. However, NO inhibition of hepatic phosphoenolpyruvate carboxykinase (PEPCK) activity leading to reduction of gluconeogenesis is also known (5). Therefore, we thought it is possible that nipradilol suppresses hepatic gluconeogenesis through inhibition of PEPCK activity. If so, this would be a novel antidiabetic action of nipradilol that will be of potential

*Corresponding author. FAX: +81-263-37-2710

E-mail: keishi@hsp.md.shinshu-u.ac.jp clinical benefit.

In the present study, we explored such a possibility by evaluating the direct effect of nipradilol on PEPCK gene regulation using a rat hepatoma cell line, H4IIE cells (American Type Tissue Culture Collection, Rockville, MD, USA). Because expression of PEPCK gene is primarily regulated at the level of transcription (6) and glucocorti-

A)

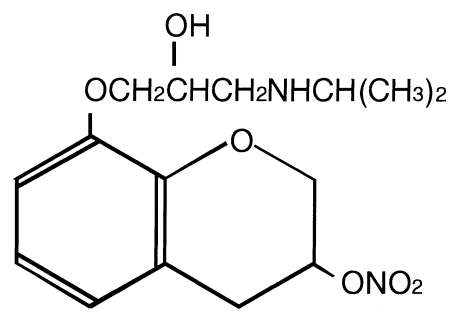

B)

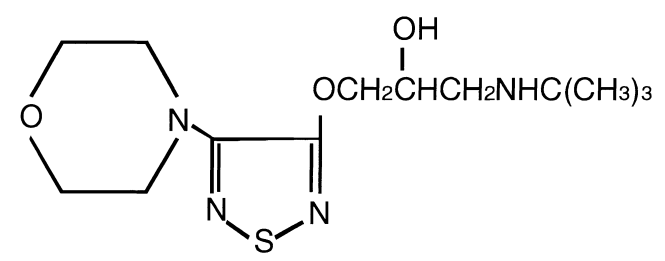

Fig. 1. Structure of nipradilol (A) and timolol (B). 
coids stimulate the PEPCK gene transcription (7), we determined whether nipradilol modifies PEPCK gene transcription in the control and the dexamethasone-treated cells.

For estimation of PEPCK gene transcription level, we employed the reporter method. A DNA of the genomic sequence from nucleotides -493 to +1 of the $5^{\prime}$ end of the human PEPCK gene was inserted into a vector with the luciferase gene to generate PEPCK luciferase (PEPCK/L) (8). PEPCK/L was then transfected into H4IIE cells maintained in Minimal Eagle Medium $\alpha$ modification ( $\alpha$ MEM) supplemented with $10 \%$ heat-inactivated FBS. The transfection was done using lipofectinamin (Gibco, Life Technologies, Gaithersburg, MD, USA) (9), as described in the manufacturers instruction. After overnight incubation, the cells were washed with and placed into serum-free $\alpha$ MEM. During the subsequent $12 \mathrm{~h}$, the cells were treated with reagents. Then, whole cell lysates were prepared for determination of luciferase activities. Luciferase assay of cell lysates was performed using commercially available luciferase assay kits (Promega, Madison, WI, USA).

As well established (7), dexamethasone (Sigma Chemical Co., St. Louis, MO, USA) increased the rate of PEPCK gene transcription 3-fold (Fig. 2A). On the other hand,
$100 \mathrm{nM}$ insulin (Sigma) slightly suppressed basal PEPCK gene transcription as previous known (6) and did not significantly alter dexamethasone-induced increase in PEPCK gene transcription (Fig. 2A). Nipradilol slightly but significantly suppressed basal PEPCK gene transcription. Additionally, treatment of the cells with nipradilol strongly restrained dexamethasone-induced increase in PEPCK gene transcription (Fig. 2B). In contrast, the same concentration of timolol (Sigma), a $\beta$-blocker without a nitroxy moiety (Fig. 1B) (10), did not significantly alter basal and dexamethasone-induced PEPCK gene transcription (Fig. 2B). On the molar basis, timolol is approximately 3 times more potent than nipradilol as a $\beta$-blocker (3). As shown in Fig. 3 , inhibitory effects of nipradilol on PEPCK gene transcription were completely eradicated by $10 \mu \mathrm{M}$ methylene blue, an inhibitor of nitric oxide-inducible guanylate cyclase (11).

It is reported that nipradilol releases $\mathrm{NO}$ and stimulates guanylate cyclase (12), and our findings agree well with such a notion. Namely, as shown in Fig. 2, suppression of PEPCK gene transcription is a unique feature of nipradilol, not shared by timolol that lacks a nitroxy molecule. In addition, methylene blue, an inhibitor of soluble guanylate
A)

control

$\triangle 100 \mathrm{nM}$ dexamethasone

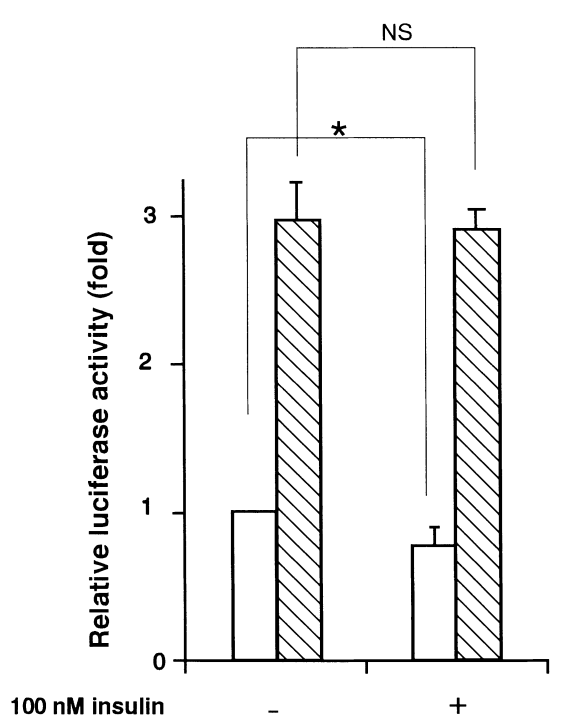

B)

control

$100 \mathrm{nM}$ dexamethasone

NS

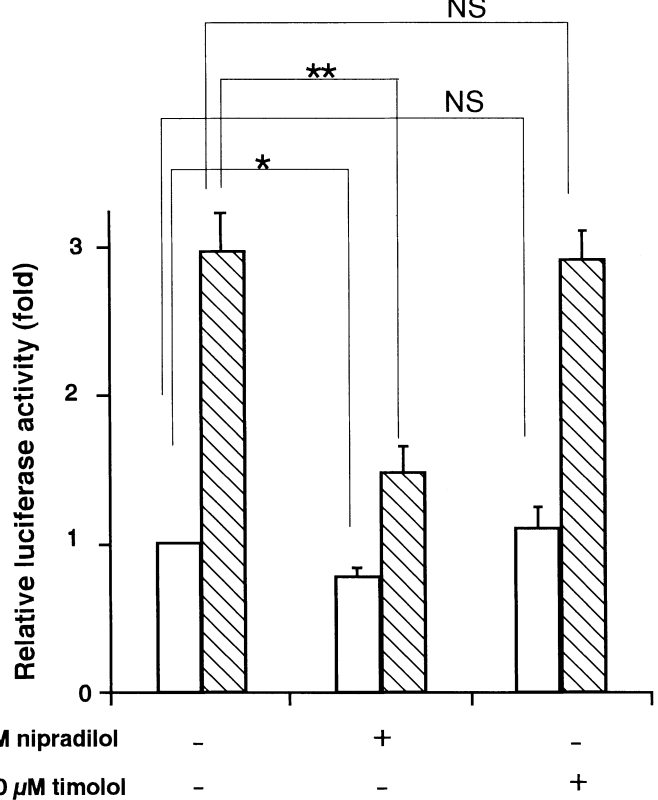

Fig. 2. Effect of insulin and $\beta$-blockers on basal and dexamethasone-stimulated PEPCK gene transcription in H4IIE cells. The data is presented as PEPCK/L activity in the test conditions divided by that in the basal (untreated) condition. Representative data from 3 independent experiments are shown. Values are means \pm S.D. $\left(\mathrm{n}=3\right.$ for each condition). $* P<0.05$, ${ }^{*} P<0.01$ (Wilcoxon's rank sum test). A) H4IIE cells were incubated with $100 \mathrm{nM}$ insulin in the presence or absence of $100 \mathrm{nM}$ dexamethasone for $12 \mathrm{~h}$. B) H4IIE cells were incubated with $100 \mu \mathrm{M}$ nipradilol or $100 \mu \mathrm{M}$ timolol in the presence or absence of $100 \mathrm{nM}$ dexamethasone for $12 \mathrm{~h}$ 
$\square$ control

$10 \mu \mathrm{M}$ methylene blue

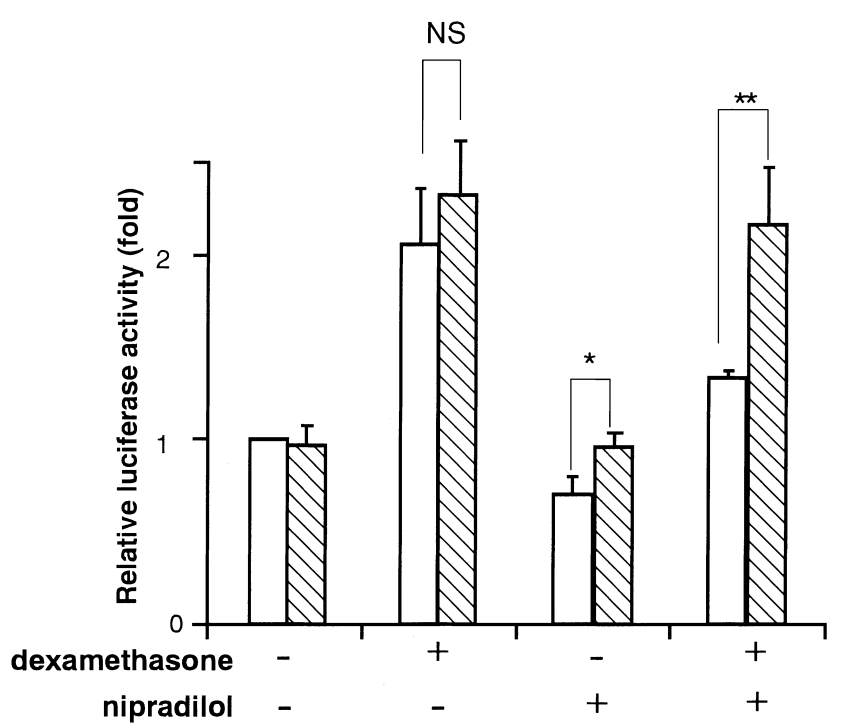

Fig. 3. Effects of methylene blue on PEPCK gene transcription. The data is presented as PEPCK/L activity in the test conditions divided by that in the basal (untreated) condition. Representative data from 3 independent experiments are shown. Values are means \pm S.D. ( $\mathrm{n}=3$ for each condition). ${ }^{*} P<0.05,{ }^{*} P<0.01$ (Wilcoxon's rank sum test). H4IIE cells were incubated with $100 \mathrm{nM}$ dexamethasone and/or $100 \mu \mathrm{M}$ nipradilol in the presence or absence of $10 \mu \mathrm{M}$ methylene blue for $12 \mathrm{~h}$.

cyclase, strongly inhibited nipradilol's effects on PEPCK gene transcription.

Inhibition of glucagon-stimulated glycogenolysis by NO-induced accumulation of cyclic guanosine monophosphate was previously reported (13). This is due to stimulation of guanylate cyclase by NO. NO-induced suppression of gluconeogenesis is also reported by others (5). We here provided at least a part of the molecular basis for $\mathrm{NO}$ suppression of gluconeogenesis.

One should always bear in mind suppression of insulin secretion by $\beta$-blocker as a potential adverse effect of this line of drugs. However, nipradilol, as a NO donor, likely ameliorates insulin resistance both at the level of liver and the level of vasculature. Thus, it may be useful in the treatment of diabetic patients with hypertension or coronary insufficiency.

\section{Acknowledgments}

Nipradilol was a generous gift from the Kowa Company, Ltd. (Nagoya). PEPCK luciferase (PEPCK/L) plasmid was kindly provided by Dr. Kurt Jungermann (Institut für Biochemie und Molekuläre Zellbiologie, Germany).

\section{REFERENCES}

1 Museteanu P, Fica S and Gudovan E: New insights in steroid diabetes. Endocrinologie 29, 105 - 109 (1991)

2 Sasage H, Nakazawa M, Arakawa M and Imai S: Contribution of cyclic GMP generation to the relaxation by nipradilol in the rabbit aorta. Gen Pharmacol 26, 85 - 91 (1995)

3 Hayashi T and Iguchi A: Nipradilol: a $\beta$-adrenoceptor antagonist with nitric oxide-releasing action. Cardiovasc Drug Rev 15, $212-235$ (1998)

4 Rosen P, Ballhausen $\mathrm{T}$ and Stockklauser K: Impairment of endothelium dependent relaxation in the diabetic rat heart: mechanisms and implications. Diabetes Res Clin Pract 31, Suppl, S143 - S155 (1996)

5 Ou J, Molina L, Kim Y-M and Billiar TR: Excessive NO production does not account for inhibition of hepatic glucogenesis in endotoxemia. Am J Physiol 271, G621 - G628 (1996)

6 Gabbay RA, Sutherland C, Gnudi L, Kahn BB, O'Brien RM, Granner DK and Flier JS: Insulin regulation of phosphoenolpyruvate carboxykinase gene expression does not require activation of the Ras/mitogen-activated protein kinase signaling pathway. J Biol Chem 271, 1890 - 1897 (1996)

7 Friedman JE, Sun Y, Ishizuka T, Farrell CJ, McCormack SE, Herron LM, Hakimi P, Lechner P and Yun JS: Phosphoenolpyruvate carboxykinase (GTP) gene transcription and hyperglycemia are regulated by glucocorticoids in genetically obese $\mathrm{db} / \mathrm{db}$ transgenic mice. J Biol Chem 272, 31475 - 31481 (1997)

8 Christ B, Nath A and Jungermann K: Mechanism of the impairment of the glucagon-stimulated phosphoenolpyruvate carboxykinase gene expression by interleukin- 6 in rat hepatocytes: inhibition of the increase in cyclic 3 ',5' adenosine monophosphate and the downstream cyclic $3^{\prime}, 5^{\prime}$ adenosine monophosphate action. Hepatology 26, 73 - 80 (1997)

9 Raha A, Joyce T, Gusky S and Bresnick E: Glycine $N$-methyltransferase is a mediator of cytochrome P4501A1 gene expression. Arch Biochem Biophys 322, 395 - 404 (1995)

10 Prichard $\mathrm{BN}$ : $\beta$-Adrenergic receptor blockade in hypertension, past, present and future. Br J Clin Pharrmacol 5, 379-399 (1978)

11 Maruna P, Masek Z and Schreiber V: Methylene blue inhibition of oestradiol-induced increase of ceruloplasmin serum levels in rats. Physiol Res 43, 219 - 221 (1994)

12 Okamura T, Kitamura Y, Uchiyama M, Toda M, Ayajiki K and Toda N: Canine retinal arterial and arteriolar dilatation induced by nipradilol, a possible glaucoma therapeutic. Pharmacology 53, $302-310$ (1998)

13 Ceppi ED and Titheradge MA: The importance of nitric oxide in the cytokine-induced inhibition of glucose formation by cultured hepatocytes incubated with insulin, dexamethasone, and glucagon. Arch Biochem Biophys 349, 167 - 174 (1998) 